\title{
Editorial
}

\section{Translational Molecular Imaging Computing: Advances in Theories and Applications}

\author{
Jinchao Feng, ${ }^{1}$ Wenxiang Cong, ${ }^{2}$ Kuangyu Shi, ${ }^{3}$ Shouping Zhu, ${ }^{4}$ and Jun Zhang ${ }^{5}$ \\ ${ }^{1}$ Faculty of Information Technology, Beijing University of Technology, Beijing 100124, China \\ ${ }^{2}$ Department of Biomedical Engineering, Rensselaer Polytechnic Institute, Troy, NY 12180, USA \\ ${ }^{3}$ Department of Nuclear Medicine, Technical University of Munich, 81675 Munich, Germany \\ ${ }^{4}$ School of Life Science and Technology, Xidian University, Xian 710071, China \\ ${ }^{5}$ Department of Radiology, University of North Carolina at Chapel Hill, Chapel Hill, NC 27599, USA
}

Correspondence should be addressed to Jinchao Feng; fengjc@bjut.edu.cn

Received 27 November 2016; Accepted 27 November 2016

Copyright (C) 2016 Jinchao Feng et al. This is an open access article distributed under the Creative Commons Attribution License, which permits unrestricted use, distribution, and reproduction in any medium, provided the original work is properly cited.

Molecular imaging is capable of revealing cellular and molecular features of organism and disease in vivo, meeting the increasing demands in the noninvasive understanding of biological processes. Computational technologies are essential for the development of cutting-edge molecular imaging. In the past, the advancement of molecular imaging computing has been well recognized and continuously extends the application potential of molecular imaging.

The papers selected for this special issue represent recent progress in molecular imaging computing, including applications, high-performance computing technologies, method and algorithm improvement, and review. All of these papers not only provide novel ideas and state-of-the-art technologies in the field but also facilitate future research for translational molecular imaging.

Computed tomography (CT) is one of the commonly used imaging techniques. Now, the use of CT has increased rapidly. However, it involves radiation doses during a CT exam, which are harmful to the patient. When the radiation dose decreases, the relative noise in CT images will increase, which deteriorate the image quality. Therefore, how to reduce CT scanning dose of patients while maintaining the same image quality is a challenging problem. L. Deng et al. improved a total variation minimization method to enhance the image quality of CT by incorporating prior images. $\mathrm{M}$. Li et al. presented an improved smoothed $l_{0}$-norm regularization method to suppress artifacts and to obtain better edge preservation in reconstructed images.
Optical tomography (OT) is one of the most sensitive molecular imaging techniques and is especially suited for preclinical studies. Systematic reviews of OT will improve researchers' understanding and skills in utilizing the technique. B. Zhu and A. Godavarty reviewed technical aspects of fluorescence-enhanced optical tomography (also called fluorescence molecular tomography, FMT) including the principal, measurement approaches, forward model, and inverse problem.

B. Zhu and A. Godavarty mentioned that the inverse problem of FMT is severally ill-posed and underdetermined due to nonuniqueness and a limited number of measurements. To alleviate the ill-posedness of FMT, H. Yi et al. presented a feasible region extraction strategy based on a double mesh. To increase computational efficiency, D. Chen et al. developed a sparsity-constrained preconditioned Kaczmarz reconstruction method. To improve the image quality of FMT, H. Zhang et al. developed a reconstruction method by minimizing the difference between $L_{1}$ and $L_{2}$ norms (i.e., $L_{1-2}$-norm).

Cherenkov luminescence imaging (CLI) is an emerging imaging modality, which captures visible photons emitted by Cherenkov radiation labeled with $\beta$-emitting radionuclides using widely available in vivo optical imaging systems. In other words, CLI uses optical means to provide information of medical radionuclides used in nuclear imaging based on Cerenkov radiation. However, the exceptionally weak Cerenkov luminescence from Cerenkov radiation is susceptible to lots of impulse noises. In the paper contributed 
by $\mathrm{X}$. Cao et al., a temporal median filter is proposed to remove this kind of impulse noises. Results of in vivo experiments demonstrated that the temporal median method can effectively remove random pulse noises induced by gamma radiation and achieve a robust CLI image.

The image resolution of pure OT or CLT is relatively low because of the high diffusion of photons in biological tissues. To improve image resolution, a new hybrid imaging modality, $\mathrm{X}$-ray luminescence computed tomography (XLCT), has been developed. XLCT utilizes X-ray luminescent nanophosphors (NPs) as imaging probes. NPs can be excited with a pencil, fan, or cone beam of X-rays. Cone beam XLCT can realize fast XLCT with relatively low scanning time compared with pencil beam XLCT. However, the reconstruction of cone beam XLCT is also an ill-posed problem. To alleviate the illposedness of XLCT, D. Chen et al. developed a hybrid reconstruction algorithm with KA-FEM method. In vivo mouse experiment was used to evaluate the feasibility of the method.

Multimodality molecular imaging is now playing an important role in preclinical and clinical research, which utilizes the strengths of different modalities and yields a hybrid imaging platform with benefits superior to those of any of its individual components, considered alone. In the paper contributed by Y. Liu et al., a dual-modality imaging system which combines multispectral photoacoustic computed tomography and ultrasound computed tomography was developed to reconstruct functional and structural information of human finger joint systems. Phantom and in vivo results illustrated that the bones, the blood vessels, and the subcutaneous tissues could be reconstructed using the dualmodality system.

Jinchao Feng

Wenxiang Cong

Kuangyu Shi

Shouping Zhu

Jun Zhang 

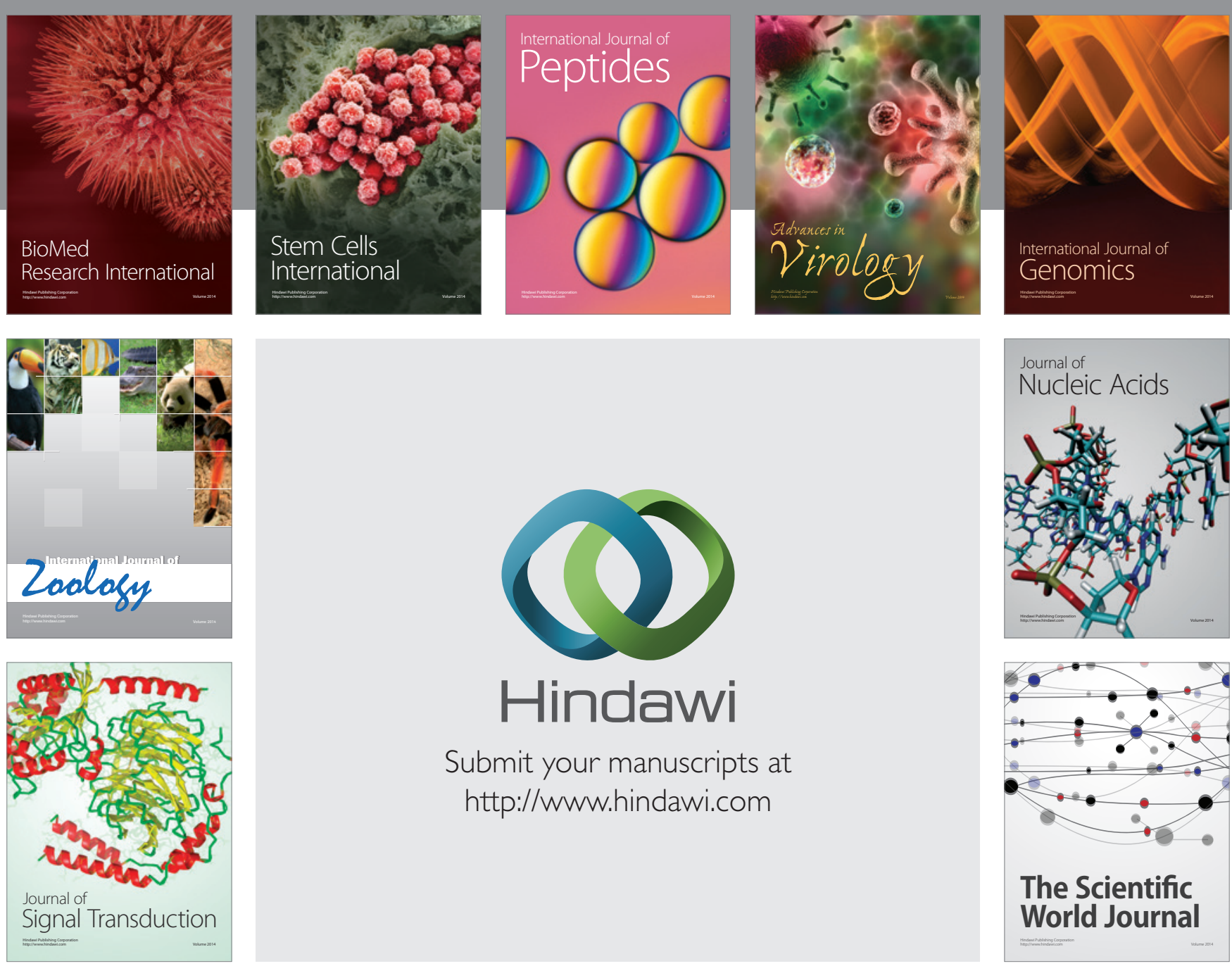

Submit your manuscripts at

http://www.hindawi.com
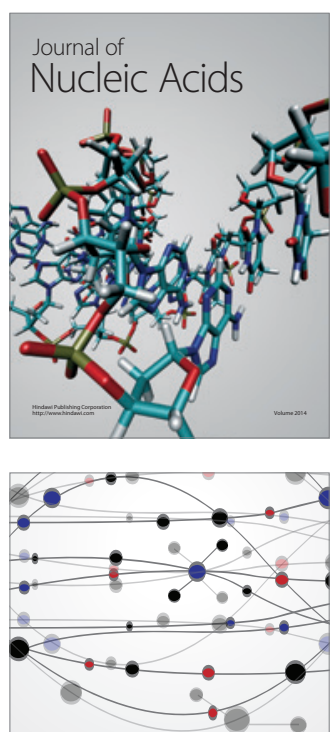

The Scientific World Journal
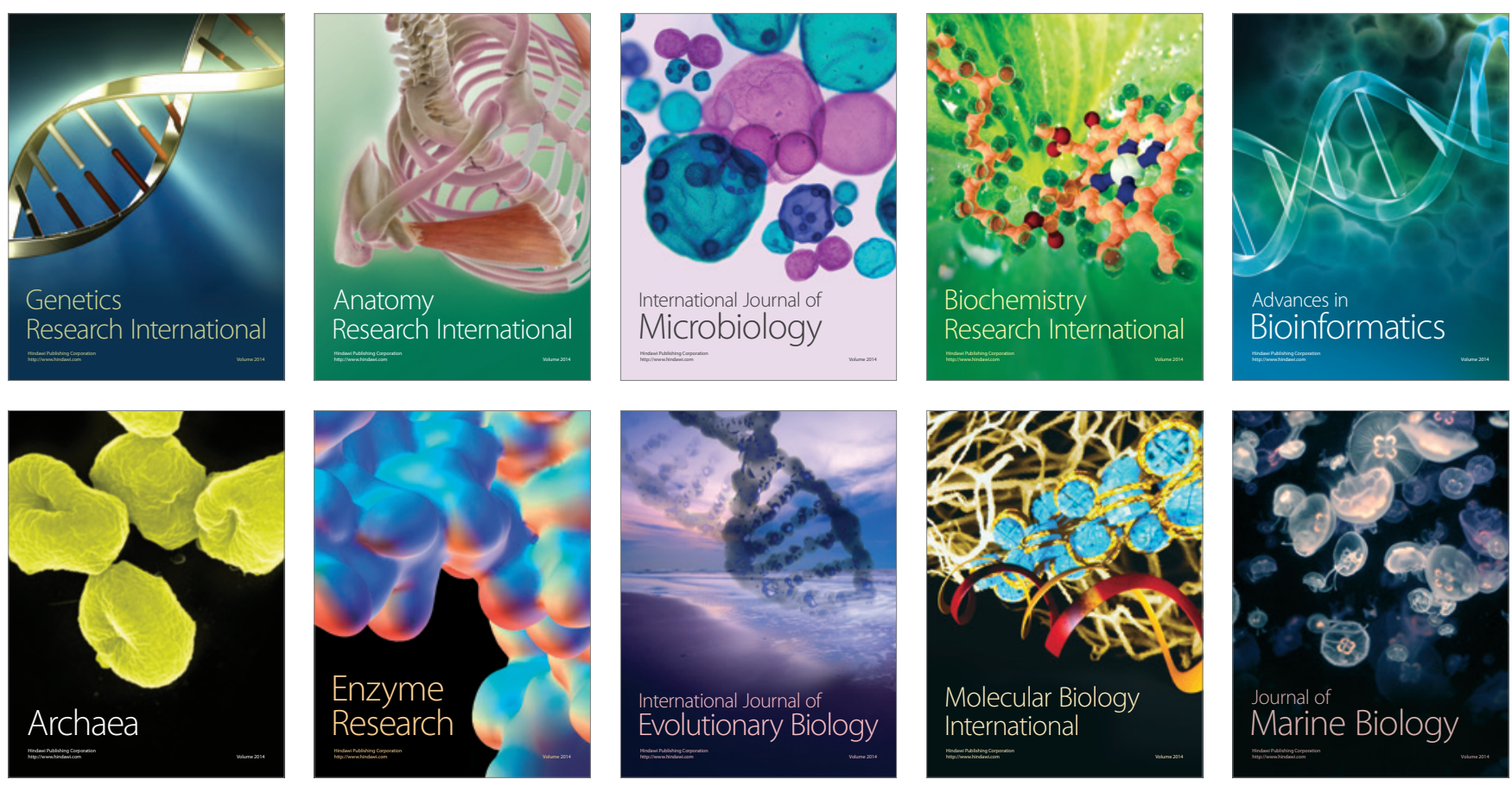